\title{
sciendo
}

Int. J. of Applied Mechanics and Engineering, 2021, vol.26, No.3, pp.163-176

DOI: 10.2478/ijame-2021-0042

\section{NEW ITERATIVE METHOD OF SOLVING NONLINEAR EQUATIONS IN FLUID MECHANICS}

\author{
M. PALIIVETS* \\ Department of Information Technology in the Agro-Industrial Complex \\ Russian State Agrarian University - Moscow Timiryazev Agricultural Academy, Moscow, RUSSIA \\ E-mail: paliivetsmax@rambler.ru, paliivets@rgau-msha.ru \\ E. ANDREEV \\ Department of Agricultural Construction and Real Estate Expertise, \\ Russian State Agrarian University - Moscow Timiryazev Agricultural Academy, Moscow, RUSSIA
}

A. BAKSHTANIN

Department of Integrated Water Management and Hydraulics, Russian State Agrarian University - Moscow Timiryazev Agricultural Academy, Moscow, RUSSIA

D. Benin and V. SNEZHKO

Department of Information Technology in the Agro-Industrial Complex, Russian State Agrarian University - Moscow Timiryazev Agricultural Academy, Moscow, RUSSIA

\begin{abstract}
This paper presents the results of applying a new iterative method to linear and nonlinear fractional partial differential equations in fluid mechanics. A numerical analysis was performed to find an exact solution of the fractional wave equation and fractional Burgers' equation, as well as an approximate solution of fractional $\mathrm{KdV}$ equation and fractional Boussinesq equation. Fractional derivatives of the order $\alpha$ are described using Caputo's definition with $0<\alpha \leq 1$ or $1<\alpha \leq 2$. A comparative analysis of the results obtained using a new iterative method with those obtained by the Adomian decomposition method showed the first method to be more efficient and simple, providing accurate results in fewer computational operations. Given its flexibility and ability to solve nonlinear equations, the iterative method can be used to solve more complex linear and nonlinear fractional partial differential equations.
\end{abstract}

Keywords: Adomian decomposition method (ADM), earthquake, filtration, fluid mechanics, iterative method, nonlinear fractional partial differential equations, porous medium.

\section{Introduction}

Fractional partial differential equations (FPDEs) are useful tools to model many physical phenomena such as nonlinear seismic oscillations, hydrodynamic movement, decay laws, diffusion processes, electrostatics, electrodynamics, elasticity [1-4]. For example, FPDEs have been used to model filtration flow in a porous medium [5]. Solving FPDEs is quite a complicated process, which requires highly complex mathematical calculations and solution methods. Most modern computer programs are not suitable or require extra calculations to obtain accurate FPDEs solutions in terms of complex elementary functions. Therefore, an efficient and reliable numerical algorithm for solving such equations is highly relevant [6-8]. Furthermore, the interest in developing simple and accessible numerical methods in mathematics, physics and engineering has increased considerably in recent years. Thus, a number of works have studied and solved various FPDEs:

\footnotetext{
${ }^{*}$ To whom correspondence should be addressed
} 
space-time fractional diffusion-wave equation [9], fractional advection dispersion equation [10], fractional telegraph equation [11], fractional Korteweg-de Vries (KdV) equation [12], and linear inhomogeneous FPDEs [13].

Fractional computation is a theory of integrals and derivatives of an arbitrary order that unites and generalizes concepts of integral differentiation and $n$-fold integration [14]. The advantage of fractional computational methods for modeling physical and mathematical phenomena is their nonlocality [15]. Given that the differential operators of integer order and integral operators of integer order are local, then the differential operators of fractional order and the integral operators of fractional order are non-local. It means that the next condition of the system is dependent not only on its present but also on all its historical conditions. This feature of fractional computing is an excellent tool for describing the memory and inherited properties of various physical and technical processes [16].

Series expansions are an important tool in numerical computations for quick assessments when calculating functions, integrals, or derivatives [17]. The expansion methods allow finding solutions to FPDEs through a series expansion, providing some advantages in multivariate solutions for FPDEs. One of such methods is the Adomian Decomposition Method (ADM) [18], which is used to provide an analytical approximation to linear and nonlinear tasks. This approach also provides direct and visible symbolic terms for analytical solutions and numerical approximations of linear and nonlinear differential equations with no linearization or discretization. The new iterative method (IM) is also an analytical and numerical approximation of solutions to linear and nonlinear tasks $[19,20]$. The iterative method $[21,22]$ has been successfully applied to a variety of linear and nonlinear equations, such as algebraic equations, integral equations, integro-differential equations, ordinary and partial differential equations of integral and fractional order, and systems of equations as well. Contrary to the ADM, the IM is fairly simple to understand and easy to implement in various computer programs, ensuring better results [23]. The IM advantage is independence from rounding errors, as it requires no linearization, perturbations, sampling, and a large amount of computer memory. The IM presents the solution as a convergent fractional series with computable elements. This method calculates the coefficients of a power series using a chain of algebraic equations of one or more variables.

The study aims to extend the application of the new iterative method for obtaining analytical solutions of FPDEs in fluid mechanics using as examples the wave equation, the Burgers' equation, the KdV equation, and the Boussinesq equation. The results obtained with the IM are compared with those obtained with the ADM to confirm the efficacy and simplicity of the IM for the equations listed and other similar equations. In this work, FPDEs were derived from the corresponding integer-order equations by replacing the first- or second-order time derivative with a fractional derivative of order $\alpha$ with $0<\alpha \leq 1$ or $1<\alpha \leq 2$ according to Caputo [24].

\section{Methods}

First, the fundamental definitions and properties of fractional calculation theory shall be introduced [25]. Definition 1. A real function $f(t), t>0$ is in the space $C_{\mu}, \mu \in R$, if such a real number $p(>\mu)$ exists that $f(t)=t^{p} f_{l}(t)$, where $f_{l}(t) \in C[0, \infty]$, and is in space $C_{\mu}^{m}$, when and only when $f^{(m)} \in C_{\mu}, m \in N$.

Definition 2. The Riemann-Liouville fractional integral of order $\alpha \geq 0$ for a function $f \in C_{\mu}, \mu \geq-1$ is defined as:

$$
\begin{aligned}
& I^{\alpha} f(t)=\frac{1}{\Gamma(\alpha)} \int_{0}^{t}(t-\tau)^{\alpha-1} f(\tau) d \tau, \quad \alpha>0, t>0, \\
& I^{0} f(t)=f(t) .
\end{aligned}
$$

The properties of the $I^{\alpha}$ operator are described in detail in $[25,26]$. 
For $f \in C_{\mu}, \quad \mu \geq-1, \quad \alpha, \beta \geq 0$ and $\quad \gamma>-1$, the following equations can be obtained:

$$
\begin{aligned}
& I^{\alpha} I^{\beta} f(t)=I^{\alpha+\beta} f(t), \\
& I^{\alpha} I^{\beta} f(t)=I^{\beta} I^{\alpha} f(t), \\
& I^{\alpha} t^{\gamma}=\frac{\Gamma(\gamma+1)}{\Gamma(\alpha+\gamma+1)} t^{\alpha+\gamma} .
\end{aligned}
$$

Definition 3. The Riemann-Liouville derivative presents certain disadvantages when it comes to modeling real phenomena using equations with fractional derivatives. For this purpose, a modified fractionaldifferential operator $D^{\alpha}$ following the Caputo viscous elasticity theory [24] shall be introduced, according to which the fractional derivative of $f(t)$ is defined as:

$$
\begin{aligned}
& D^{\alpha} f(t)=I^{m-\alpha} D^{m} f(t)=\frac{1}{\Gamma(m-\alpha)} \int_{0}^{t}(t-\tau)^{m-\alpha-1} f(t) d t \\
& \text { for } \quad m-1\langle\alpha \leq m, m \in N, t\rangle 0, \quad f \in C_{-1}^{m}
\end{aligned}
$$

Two basic properties will be required in this work.

Lemma 1. If $m-1<\alpha \leq m, \quad m \in N$ and $f \in C_{\mu}^{m}$, then

$$
\begin{aligned}
& D^{\alpha} I^{\alpha} f(t)=f(t) \\
& I^{\alpha} D^{\alpha} f(t)=f(t)-\sum_{k=0}^{m-1} f^{(k)}\left(0^{+}\right) \frac{t^{k}}{k !}, \quad t>0 .
\end{aligned}
$$

The Caputo fractional derivative is used here since it allows including the traditional baseline and boundary conditions in the problem formulation [27]. This paper considers one-dimensional linear inhomogeneous equations in fractional partial derivatives in fluid mechanics, where the unknown function is a causal function of time, converting into zero at $(t<0)$.

Definition 4. For $m$ to be the smallest integral exceeding $\alpha$, the Caputo fractional time derivative of order $\alpha>0$ is defined as:

$$
D^{\alpha} U(x, t)=\frac{\partial^{2} U}{\partial t^{2}}=\left\{\begin{array}{l}
\frac{1}{\Gamma(m-\alpha)} \int_{0}^{t}(t-\tau)^{m-\alpha-1} \frac{\partial^{m}}{\partial \tau^{m}} U(x, \tau) d \tau, \quad m-1<\alpha \leq m, \\
\frac{\partial^{m}}{\partial t^{m}} U(x, t), \quad \alpha=m, \quad m \in N .
\end{array}\right.
$$

More information on the mathematical properties of fractional derivatives and integrals is provided in [28, 29]. 


\subsection{New iterative method}

The iterative method is applicable to all kinds of differential equations outlined in [19, 20], including fractional-differential equations over time:

$$
\frac{\partial^{\alpha}}{\partial t^{\alpha}} U(x, t)=R[x] U(x, t)+q(x, t), \quad t>0, \quad x \in R
$$

where $R[x]$ is a differential operator in $x$ under baseline and boundary conditions:

$$
\begin{aligned}
& U(x, 0)=f(x), \quad 0<\alpha \leq 1, \\
& U(x, t) \rightarrow 0 \text { as }|x| \rightarrow \infty, \quad t>0, \\
& U(x, 0)=f(x), \quad \frac{\partial U(x, 0)}{\partial t}=g(x), \quad 1<\alpha \leq 2, \\
& U(x, t) \rightarrow 0 \text { as }|x| \rightarrow \infty, \quad t>0,
\end{aligned}
$$

where $f(x), g(x)$, and $q(x, t)$ are continuous functions, and $\alpha$ is an order parameter of the fractional time derivative $(m-1<\alpha \leq m)$.

The corrective functional for Eq.(2.5) has the following form:

$$
\begin{aligned}
& U_{k+1}(x, t)=U_{k}(x, t)+I_{t}^{\beta}\left[\lambda\left(\frac{\partial^{\alpha} U_{k}(x, t)}{\partial t^{\alpha}}-R[x] \widetilde{U_{k}}(x, t)-q(x, t)\right)\right]= \\
& =U_{k}(x, t)+\frac{1}{\Gamma(\beta)} \int_{0}^{t}(t-\tau)^{\beta-1} \lambda(\tau)\left(\frac{\partial^{\alpha} U_{k}(x, \tau)}{\partial t^{\alpha}}-R[x] \widetilde{U_{k}}(x, \tau)-q(x, \tau)\right) d \tau
\end{aligned}
$$

where $I_{t}^{\beta}$ is the Riemann-Liouville fractional integral of order $\beta=\alpha+1-m$ over the variable $t$, and $\lambda$ is the Lagrange multiplier [30]. To determine $\lambda$, Eq.(2.8) shall be simplified as some approximation is necessary. The correction functional can be approximately expressed as follows:

$$
U_{k+l}(x, t)=U_{k}(x, t)+\int_{0}^{t}\left(\lambda(\tau)\left(\frac{\partial^{m} U_{k}(x, \tau)}{\partial t^{m}}-R[x] \widetilde{U_{k}}(x, \tau)-q(x, \tau)\right) d \tau\right) .
$$

Assuming bounded variations to the nonlinear term $R[x] U$ Eq.(2.9) shall be written in the stationary form at $\delta \widetilde{U_{k}}=0$ :

$$
\delta U_{k+1}(x, t)=\delta U_{k}(x, t)+\delta \int_{0}^{t}\left(\lambda(\tau)\left(\frac{\partial^{m} U_{k}(x, \tau)}{\partial t^{m}}-q(x, \tau)\right) d \tau\right)
$$


By solving this equation one can obtain the following Lagrange multipliers:

$$
\begin{aligned}
& \lambda=-1 \quad \text { when } \quad m=1, \\
& \lambda=\tau-t, \quad \text { when } \quad m=2 .
\end{aligned}
$$

Consequently, substituting the Lagrange multiplier in (2.8), the following iteration formula for $m=1$ shall be obtained:

$$
U_{k+1}(x, t)=U_{k}(x, t)+I_{t}^{\alpha}\left[\frac{\partial^{\alpha} U_{k}(x, t)}{\partial t^{\alpha}}-R[x] U_{k}(x, t)-q(x, t)\right] .
$$

If $m=2$, Eq.(2.8) will be as follows:

$$
U_{k+1}(x, t)=U_{k}(x, t)-\frac{\alpha-1}{\Gamma(\alpha)} \int_{0}^{t}(\tau-t)^{\alpha-2}\left(\frac{\partial^{\alpha} U_{k}(x, \tau)}{\partial t^{\alpha}}-R[x] U_{k}(x, \tau)-q(x, \tau)\right) d \tau .
$$

The iteration formula is obtained in the following form:

$$
U_{k+1}(x, t)=U_{k}(x, t)+(\alpha-1) I_{t}^{\alpha}\left[\frac{\partial^{\alpha} U_{k}(x, t)}{\partial t^{\alpha}}-R[x] U_{k}(x, t)-q(x, t)\right] .
$$

The initial approximation $U_{0}$ is chosen so that the baseline and boundary conditions of the problem are satisfied, after which the solution $U(x, t)=\lim _{k \rightarrow \infty} U_{k}(x, t)$ by the $N$-th term $U_{N}(x, t)$ is approximated. To illustrate the basic idea of iteration, a general fractional differential equation (2.5) with partial derivatives of arbitrary fractional order shall be considered:

$$
\frac{\partial^{\alpha}}{\partial t^{\alpha}} U(x, t)=L[x] U(x, t)+N[x] U(x, t)+q(x, t), \quad t>0, \quad x \in R
$$

where $L[x] U(x, t)$ is a linear operator on $x$, and $N[x] U(x, t)$ is a nonlinear operator on $x$. The method is based on applying the operator $I_{t}^{\alpha}$, which is inverse to the operator $D_{t}^{\alpha}$, to both parts of Eq.(2.15) in order to obtain an equivalent fractional-integral equation:

$$
U(x, t)=\sum_{k=0}^{m-1} \frac{\partial^{k} U}{\partial t^{k}}\left(0^{+}\right) \frac{t^{k}}{k !}+I_{t}^{\alpha}(L[x] U(x, t)+N[x] U(x, t)+q(x, t)) .
$$

\subsection{Adomian Decomposition Method (ADM)}

To solve Eq.(2.16), the Adomian decomposition method (ADM) shall be used and $U(x, t)$ shall be decomposed into an infinite component series [18]: 


$$
\begin{aligned}
& U(x, t)=\sum_{n=0}^{\infty} U_{n}(x, t), \\
& N U=\sum_{n=0}^{\infty} P_{n}
\end{aligned}
$$

where $\mathrm{P}_{\mathrm{n}}-$ Adomian polynomials. The expansion series (2.17) are substituted into both parts of Eq.(2.16):

$$
\sum_{n=0}^{\infty} U_{n}(x, t)=\sum_{k=0}^{m-1} \frac{\partial^{k} U}{\partial t^{k}}\left(0^{+}\right) \frac{t^{k}}{k !}+I_{t}^{\alpha}\left(R[x] \sum_{n=0}^{\infty} U_{n}(x, t)+\sum_{n=0}^{\infty} P_{n}+q(x, t)\right) .
$$

Linear and nonlinear parts of Eq.(2.18) shall be as follows:

$$
\begin{aligned}
& U_{0}(x, t)=\sum_{k=0}^{m-1} \frac{\partial^{k} U}{\partial t^{k}}\left(0^{+}\right) \frac{t^{k}}{k !}+I_{t}^{\alpha}(q(x, t)), \\
& U_{j+1}(x, t)=\sum_{k=0}^{m-1}+I_{t}^{\alpha}\left(L[x] U_{j}(x, t)+P_{j}\right), \quad j \geq 0
\end{aligned}
$$

where the Adomian polynomial $P_{n}$ can be calculated for all forms of nonlinearity according to [18] by the formula:

$$
P_{n}=\frac{1}{n !}\left(\frac{d^{n} N \sum_{k=0}^{n} \lambda^{k} U_{k}}{d \lambda^{n}}\right)_{\lambda=0}
$$

Using mathematical formula (2.20), the necessary number of Adomian polynomials to calculate numerical and explicit solutions was obtained. In the case of a given initial component $U_{0}$, the remaining components $U_{j}$ can be determined one by one using the previous terms. The iterative method provides a solution in the form of a rapidly converging series that can lead to an exact solution for linear differential equations and an efficient highly-accurate numerical solution for nonlinear equations.

\section{Results}

\subsection{Solving linear equations}

The efficiency of the iterative method will be compared with the ADM on the example of two fractional-linear partial differential equations. The following one-dimensional linear inhomogeneous fractional wave equation in fluid mechanics is considered:

$$
D_{x}^{\alpha} U+U_{x}=\frac{t^{\alpha+1}}{\Gamma(2-\alpha)} \sin x+t \cos x, \quad t>0, \quad x \in R, \quad 0<\alpha \leq 1
$$

under the initial condition:

$$
U(x, 0)=0
$$


The problem is solved by using the ADM, and the first components of the solution can be written in the following form:

$$
\begin{aligned}
& U_{0}(x, t)=t \sin x+\frac{t^{\alpha+1}}{\Gamma(2+\alpha)} \cos x, \\
& U_{1}(x, t)=-\frac{t^{\alpha+1}}{\Gamma(2+\alpha)} \cos x+\frac{t^{2 \alpha+1}}{\Gamma(2+2 \alpha)} \sin x, \\
& U_{2}(x, t)=-\frac{t^{2 \alpha+1}}{\Gamma(2+2 \alpha)} \sin x+\frac{t^{3 \alpha+1}}{\Gamma(2+3 \alpha)} \cos x .
\end{aligned}
$$

For a series of solutions, Eq.(3.3) can be represented in the form:

$$
\begin{aligned}
& U(x, t)=t \sin x+\frac{t^{\alpha+1}}{\Gamma(2+\alpha)} \cos x-\frac{t^{\alpha+1}}{\Gamma(2+\alpha)} \cos x+ \\
& +\frac{t^{2 \alpha+1}}{\Gamma(2+2 \alpha)} \sin x-\frac{t^{2 \alpha+1}}{\Gamma(2+2 \alpha)} \sin x+\frac{t^{3 \alpha+1}}{\Gamma(2+3 \alpha)} \cos x+\ldots
\end{aligned}
$$

As can be seen, there are background terms in Eq.(3.4), the reduction of which leads to the exact solution of Eqs. (3.1)-(3.2) in the following form:

$$
U(x, t)=t \sin x .
$$

Besides, Eqs. (3.1)-(3.2) can be solved using the new iterative method and formulas (2.12)-(2.13):

$$
U_{0}(x, t)=t \sin x+\frac{t^{\alpha+1}}{\Gamma(2+\alpha)} \cos x,
$$

and the initial values of Eqs (3.1) and (3.2) are equivalent to the following integral equation:

$$
U(x, t)=t \sin x+\frac{t^{\alpha+1}}{\Gamma(2+\alpha)} \cos x-I_{t}^{\alpha}\left[U_{x}\right] .
$$

Taking into account $\mathrm{N}(\mathrm{U})=x-I_{t}^{\alpha}\left[U_{x}\right]$, the initial components of the iterative solution of Eqs (3.1)(3.2) will be expressed as follows:

$$
\begin{aligned}
& U_{0}(x, t)=t \sin x+\frac{t^{\alpha+1}}{\Gamma(2+\alpha)} \cos x, \quad U_{1}(x, t)=-\frac{t^{\alpha+1}}{\Gamma(2+\alpha)} \cos x+\frac{t^{2 \alpha+1}}{\Gamma(2+2 \alpha)} \sin x, \\
& U_{2}(x, t)=-\frac{t^{\alpha+1}}{\Gamma(2+\alpha)}-\frac{t^{2 \alpha+1}}{\Gamma(2+2 \alpha)} \sin x+\frac{t^{3 \alpha+1}}{\Gamma(2+3 \alpha)} \cos x .
\end{aligned}
$$


For a series of solutions, Eq.(3.8) can be represented as:

$$
\begin{aligned}
& U(x, t)=t \sin x+\frac{t^{\alpha+1}}{\Gamma(2+\alpha)} \cos x-\frac{t^{\alpha+1}}{\Gamma(2+\alpha)} \cos x+ \\
& +\frac{t^{2 \alpha+1}}{\Gamma(2+2 \alpha)} \sin x-\frac{t^{2 \alpha+1}}{\Gamma(2+2 \alpha)} \sin x+\frac{t^{3 \alpha+1}}{\Gamma(2+3 \alpha)} \cos x+\ldots
\end{aligned}
$$

Removing the background terms of Eq.(3.9), the exact solution of Eqs (3.1)-(3.2) in the same form as (3.5) will be obtained. As can be seen, using two different methods results in the same solutions for the linear equation.

Considering the effectiveness of the iterative method on the example of solving a one-dimensional linear inhomogeneous Burgers' equation with partial derivatives, it follows:

$$
D_{t}^{\alpha} U+U_{x}-U_{x x}=\frac{2 t^{2-\alpha}}{\Gamma(3-\alpha)}+2 x-2, \quad t>0, \quad x \in R, \quad 1<\alpha \leq 2
$$

under the initial condition:

$$
U(x, 0)=x^{2}
$$

Using the ADM, the first components of the solution to the equation will be written as:

$$
\begin{aligned}
& U_{0}(x, t)=x^{2}+t^{2}+\frac{t^{\alpha}}{\Gamma(1+\alpha)}(2 x-2), \quad U_{1}(x, t)=-\frac{t^{\alpha}}{\Gamma(1+\alpha)}(2 x-2)-\frac{2 t^{2 \alpha}}{\Gamma(1+2 \alpha)}, \\
& U_{2}(x, t)=-\frac{2 t^{2 \alpha}}{\Gamma(1+2 \alpha)}, \quad U_{3}(x, t)=0 .
\end{aligned}
$$

Then the exact solution of Eq.(3.10) can be written in the following form:

$$
\begin{aligned}
& U(x, t)=x^{2}+t^{2}+\frac{t^{\alpha}}{\Gamma(1+\alpha)}(2 x-2)-\frac{t^{\alpha}}{\Gamma(1+\alpha)}(2 x-2)+ \\
& -\frac{2 t^{2 \alpha}}{\Gamma(1+2 \alpha)}+\frac{2 t^{2 \alpha}}{\Gamma(1+2 \alpha)}=x^{2}+t^{2} .
\end{aligned}
$$

Applying a new iterative method to Eqs (2.12) and (2.13), the solution of Eqs (3.10)-(3.11) is equivalent to an integral equation:

$$
U(x, t)=x^{2}+t^{2}+\frac{t^{\alpha}}{\Gamma(1+\alpha)}(2 x-2)+I_{t}^{\alpha}\left[U_{x}-U_{x x}\right]
$$


Assuming that $\mathrm{N}(\mathrm{U})=-I_{t}^{\alpha}\left[U_{x}-U_{x x}\right]$, the initial components of the iterative solution of Eqs (3.10)-(3.11) is as follows:

$$
\begin{aligned}
& U_{0}(x, t)=x^{2}+t^{2}+\frac{t^{\alpha}}{\Gamma(1+\alpha)}(2 x-2), \quad U_{1}(x, t)=-\frac{t^{\alpha}}{\Gamma(1+\alpha)}(2 x-2)-\frac{2 t^{2 \alpha}}{\Gamma(1+2 \alpha)}, \\
& U_{2}(x, t)=-\frac{2 t^{2 \alpha}}{\Gamma(1+2 \alpha)}, \quad U_{3}(x, t)=0 .
\end{aligned}
$$

As can be seen, Eq.(3.15) is identical to Eq.(3.12), and if expressing the solution in a general form, the exact solution of Eqs (3.10)-(3.11) will be as (3.13), which indicates that both methods are simple and practical.

\subsection{Solution of nonlinear equations}

Only approximate solutions can be obtained for solving nonlinear equations since no exact solution exists. The two techniques were compared to obtain approximate solutions of the nonlinear KdV equation and the Boussinesq time-fractional partial differential equation.

Considering the nonlinear $\mathrm{KdV}$ equation in the form:

$$
D_{t}^{\alpha} U+6 U U_{x}+U_{x x x}=0, \quad t>0, \quad x \in R, \quad 1<\alpha \leq 2,
$$

under the initial condition:

$$
U(x, 0)=\frac{1}{2} \operatorname{sech}^{2}\left(\frac{x}{2}\right)
$$

Using the ADM, the approximate solution of Eqs (3.16)-(3.17) will be as follows:

$$
U(x, t)=f_{0}(x)+f_{1}(x) \frac{t^{\alpha}}{\Gamma(1+\alpha)}+f_{2}(x) \frac{t^{2 \alpha}}{\Gamma(1+2 \alpha)}+f_{3}(x) \frac{t^{3 \alpha}}{\Gamma(1+3 \alpha)}+\ldots
$$

where

$$
\begin{aligned}
& f_{0}(x)=\frac{1}{2} \operatorname{sech}^{2}\left(\frac{x}{2}\right), \quad f_{1}(x)=-6 f_{0} f_{0}^{\prime}-f_{0}^{\prime \prime \prime}, \\
& f_{2}(x)=-6 f_{1} f_{0}^{\prime}-6 f_{0} f_{1}^{\prime}-f_{1}^{\prime \prime \prime}, \quad f_{3}(x)=-6 f_{2} f_{0}^{\prime}-6 f_{1} f_{1}^{\prime} \frac{\Gamma(2 \alpha+1)}{\Gamma(\alpha+1)^{2}}--6 f_{0} f_{2}^{\prime}-f_{2}^{\prime \prime \prime} .
\end{aligned}
$$

Using the new iterative method with formulas (2.12) and (2.13), the solution of Eqs (3.16)-(3.17) is equivalent to an integral equation:

$$
U(x, t)=\frac{1}{2} \operatorname{sech}^{2}\left(\frac{x}{2}\right)-I_{t}^{\alpha}\left[6 U U_{x}+U_{x x x}\right]
$$


Taking into account $\mathrm{N}(\mathrm{U})=-I_{t}^{\alpha}\left[6 U U_{x}+U_{x x x}\right]$, the initial components of the iterative solution of the equations will be expressed as:

$$
\begin{aligned}
& U_{0}(x, t)=f_{0}(x), \quad U_{1}(x, t)=f_{0}(x)+f_{1}(x) \frac{t^{\alpha}}{\Gamma(1+\alpha)}, \\
& U_{2}(x, t)=f_{0}(x)+f_{l}(x) \frac{t^{\alpha}}{\Gamma(1+\alpha)}+f_{2}(x) \frac{t^{2 \alpha}}{\Gamma(1+2 \alpha)}-66 f_{1} f_{1}^{\prime} \frac{\Gamma(2 \alpha+1)}{\Gamma(\alpha+1)^{2}} \frac{t^{3 \alpha}}{\Gamma(1+3 \alpha)} .
\end{aligned}
$$

Then, approximate solution of Eqs (3.16) can be written in a general form, which corresponds to formula (3.18) obtained by the ADM. It follows that both methods provide the same approximate solution of the KdV equation.

Considering the nonlinear Boussinesq equation with partial derivatives over time, it follows:

$$
D_{t}^{\alpha} U+\left(U^{2}\right)_{x x}+\left(U^{2}\right)_{x x x x}=0, \quad t>0, \quad x \in R, \quad 1<\alpha \leq 2,
$$

under the initial condition:

$$
U(x, 0)=\frac{4}{3} \operatorname{sech}^{2}\left(\frac{x}{4}\right), \quad U_{t}(x, 0)=-\frac{1}{3} \sinh \left(\frac{x}{2}\right)
$$

Using the ADM, the components of the approximate solution will be the following:

$$
\begin{aligned}
& U_{0}(x, t)=\frac{4}{3} \operatorname{sech}^{2}\left(\frac{x}{4}\right)+\frac{1}{3} \sinh \left(\frac{x}{2}\right) t, \\
& U_{j+1}(x, t)=-I^{\alpha}\left[\left(P_{j}\right)_{x x}-\left(P_{j}\right)_{x x x x}\right], \quad j \geq 0,
\end{aligned}
$$

where $\mathrm{Pj}$ - the Adomian polynomials of the nonlinearity $U^{2}$, which are calculated by the formulas:

$$
\begin{aligned}
& P_{0}(x, t)=U_{0}^{2}, \quad P_{1}(x, t)=2 U_{0} U_{1}, \quad P_{2}(x, t)=2 U_{0} U_{2}+U_{1}^{2}, \\
& P_{3}(x, t)=2 U_{0} U_{3}+2 U_{1} U_{2}, \quad P_{4}(x, t)=2 U_{0} U_{4}+2 U_{l} U_{3}+U_{2}^{2} .
\end{aligned}
$$

An approximate fourth-order solution for Eq.(3.21) is as follows:

$$
\begin{aligned}
& U(x, t)=\frac{4}{3} \operatorname{sech}^{2}\left(\frac{x}{4}\right)+\frac{1}{3} \sinh \left(\frac{x}{2}\right) t+\frac{1}{2(3)} \cosh \left(\frac{x}{2}\right) \frac{t^{\alpha}}{\Gamma(1+\alpha)}+ \\
& -\frac{1}{2^{2}(3)} \sinh \left(\frac{x}{2}\right) \frac{t^{\alpha+1}}{\Gamma(2+\alpha)}+\frac{1}{2^{3}(3)} \cosh \left(\frac{x}{2}\right) \frac{t^{2 \alpha}}{\Gamma(1+2 \alpha)}+ \\
& -\frac{1}{2^{4}(3)} \sinh \left(\frac{x}{2}\right) \frac{t^{2 \alpha+1}}{\Gamma(2+2 \alpha)}+\frac{1}{2^{5}(3)} \cosh \left(\frac{x}{2}\right) \frac{t^{3 \alpha}}{\Gamma(1+3 \alpha)}-\frac{1}{2^{6}(3)} \sinh \left(\frac{x}{2}\right) \frac{t^{3 \alpha+1}}{\Gamma(2+3 \alpha)}
\end{aligned}
$$


According to the iterative method and Eq.(2.12), the iterative formula for the fractional time Boussinesq equation is as follows:

$$
U(x, t)=\frac{4}{3} \operatorname{sech}^{2}\left(\frac{x}{4}\right)+\frac{1}{3} \sinh \left(\frac{x}{2}\right) t-(\alpha-1) I_{t}^{\alpha}\left[\left(U^{2}\right)_{x x}+\left(U^{2}\right)_{x x x x}\right] .
$$

Assuming that $\mathrm{N}(\mathrm{U})=-(\alpha-1) I_{t}^{\alpha}\left[\left(U^{2}\right)_{x x}+\left(U^{2}\right)_{x x x x}\right]$, a fourth-order approximate solution for the timefractional equation can be obtained:

$$
\begin{aligned}
& U(x, t)=\frac{4}{3} \operatorname{sech}^{2}\left(\frac{x}{4}\right)+\frac{1}{3} \sinh \left(\frac{x}{2}\right) t+\frac{1}{2(3)} \cosh \left(\frac{x}{2}\right) \frac{t^{\alpha}}{\Gamma(1+\alpha)}+ \\
& -\frac{1}{2^{2}(3)} \sinh \left(\frac{x}{2}\right) \frac{t^{\alpha+1}}{\Gamma(2+\alpha)}+\frac{1}{2^{3}(3)} \cosh \left(\frac{x}{2}\right) \frac{t^{2 \alpha}}{\Gamma(1+2 \alpha)}+ \\
& -\frac{1}{2^{4}(3)} \sinh \left(\frac{x}{2}\right) \frac{t^{2 \alpha+1}}{\Gamma(2+2 \alpha)}+\frac{1}{2^{5}(3)} \cosh \left(\frac{x}{2}\right) \frac{t^{3 \alpha}}{\Gamma(1+3 \alpha)}-\frac{1}{2^{6}(3)} \sinh \left(\frac{x}{2}\right) \frac{t^{3 \alpha+1}}{\Gamma(2+3 \alpha)}
\end{aligned}
$$

Hence, at $\alpha=2$, the approximate solution can be written as:

$$
\begin{aligned}
& U(x, t)=\frac{2}{3}\left[\cosh \left(\frac{x}{2}\right)\left(1+\frac{1}{2^{2}} \frac{t^{2}}{2 !}+\frac{1}{2^{4}} \frac{t^{4}}{4 !}+\ldots\right)-1\right]+ \\
& -\frac{2}{3} \sinh \left(\frac{x}{2}\right)\left[\frac{1}{2} t+\frac{1}{2^{3}} \frac{t^{3}}{3 !}+\frac{1}{2^{5}} \frac{t^{5}}{5 !}+\ldots\right]
\end{aligned}
$$

which converges to the exact solution of Eq.(3.21) when $\alpha=2$ :

$$
U(x, t)=\frac{4}{3} \sinh ^{2}\left(\frac{1}{4}(x-t)\right)
$$

As can be seen from the results for nonlinear equations, the iterative method has evident advantages due to the need to calculate the Adomian polynomials, which is much easier and more convenient for the calculation procedure compared to ADM.

\section{Discussion}

Similar results were obtained also in other studies. In [30], the authors used the IM to solve linear space FPDEs with given constraint conditions based on the generalized Taylor series formula. The results of analysis and testing showed that the proposed method is highly effective and accurate in solving equations with different constraint conditions. Numerical and graphical results demonstrate high reliability and efficiency of the proposed IM algorithm. In another study [31], the authors analyzed the efficiency of a new IM free of the second derivative to solve nonlinear equations for a photovoltaic cell. The new IM represents an improvement in the convergence of Newton's algorithm to obtain minimum iterations. The results showed that the errors obtained are absolute values, and meaningful approximations using Newton's algorithm require ten iterations of estimates, whereas the new IM requires only eight estimates. This indicates that the first IM requires nine evaluations, while the new IM requires seven evaluations of the function and one of its first derivatives with good efficiency. 
Furthermore, the use of IM shows good results for solving the nonlinear Fisher reaction-diffusion equation [32]. The authors introduced the modified equation solution using the fixed point theory and IM. The results of the geometrical construction of various iterative processes for the solution of scalar nonlinear equation [33] indicated that the geometrical interpretation of the third-order Halley and Euler methods is efficient in the case when the calculation of the second derivative is not time-consuming.

It follows from the above results that the iterative method is a simple and reliable tool for solving linear and nonlinear FPDEs, which is well consistent with the results of this work. As can be seen, the iterative method can be applied not only in fluid mechanics but also in other areas of physics and engineering, requiring fewer calculations and lower error rates in calculus.

\section{Conclusions}

This paper evaluates the effectiveness of a novel iterative method for the analytical solution of FPDEs in fluid mechanics. Using the example of wave equations, the Burgers equation, the KdV equation, and the Boussinesq equation, it was shown that the iterative method is well consistent with the results obtained using the Adomian expansion method. Besides, this method is simpler and more reliable as it does not require the calculation of Adomian polynomials for nonlinear tasks. This paper presents only a part of the iterative method applicability. In future studies, this method is expected to be used to solve more complex linear and nonlinear tasks in the fractional calculus of fluid mechanics.

\section{Nomenclature}

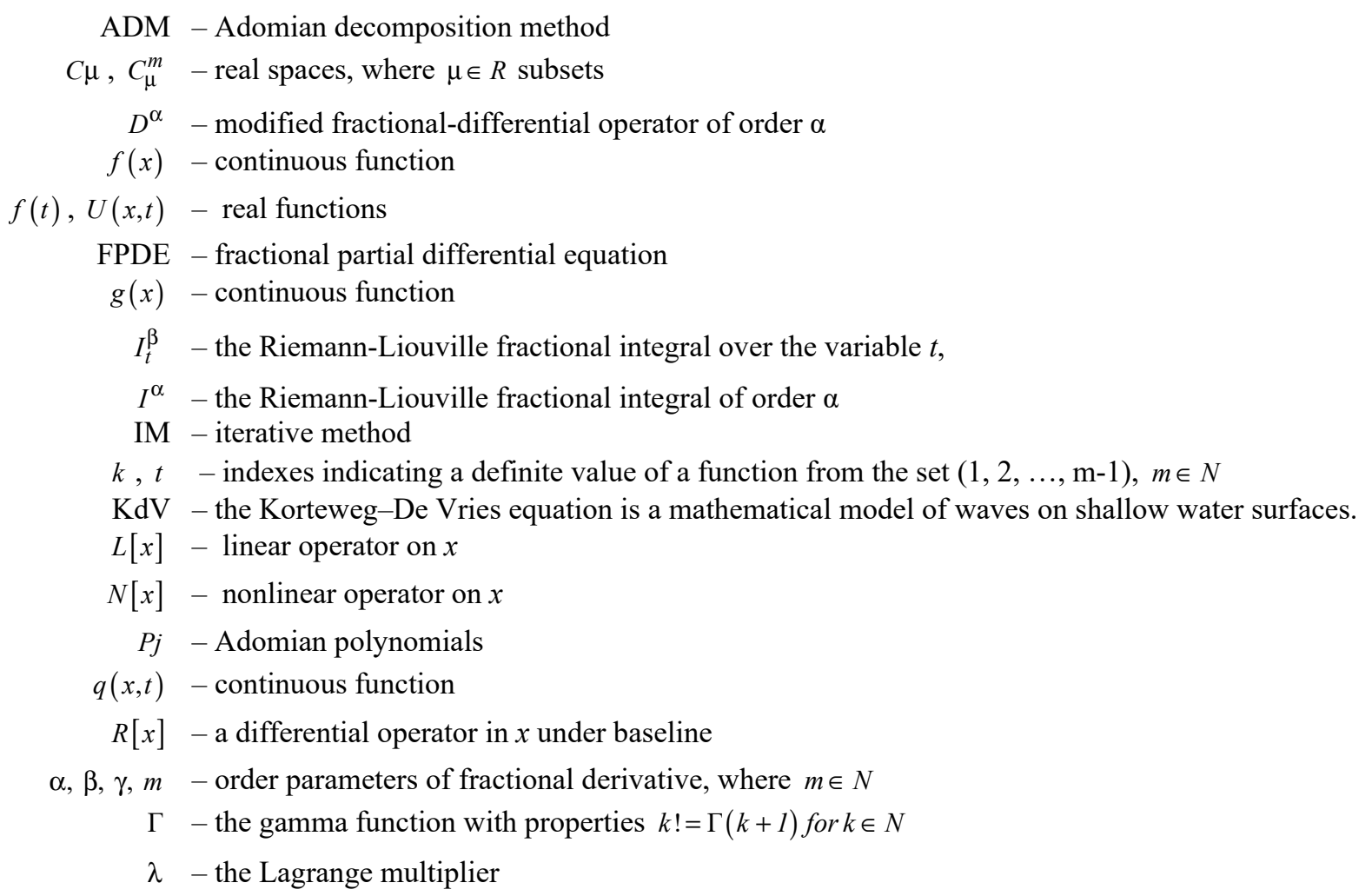

\section{Appendix}

$$
U_{x}=\frac{\partial U}{\partial x}
$$




$$
\begin{aligned}
& U_{x x}=\frac{\partial^{2} U}{\partial x^{2}} \\
& D_{t}^{\alpha} U=\frac{\partial^{\alpha} U}{\partial t^{\alpha}} \\
& U_{x x x}=\frac{\partial^{3} U}{\partial x^{3}} \\
& \left(U^{2}\right)_{x x}=\frac{\partial^{2}\left(U^{2}\right)}{\partial x^{2}} \\
& \left(U^{2}\right)_{x x x x}=\frac{\partial^{4}\left(U^{2}\right)}{\partial x^{4}}
\end{aligned}
$$

\section{References}

[1] Singh H., Kumar D. and Baleanu D. (2019): Methods of Mathematical Modelling: Fractional Differential Equations.Boca Raton: CRC Press.

[2] Shishkina E. and Sitnik S. (2020): Transmutations, Singular and Fractional Differential Equations with Applications to Mathematical Physics.-Cambridge: Academic Press.

[3] Milici C., Drăgănescu G. and Machado J.T. (2018): Introduction to Fractional Differential Equations.- Cham: Springer, vol.25.

[4] Kaplan M., Bekir A., Akbulut A. and Aksoy E. (2016): The modified simple equation method for solving some fractional-order nonlinear equations.- Pramana, vol.87, No.1, pp.1-5. https://doi.org/10.1007/s12043-016-1205-y

[5] Brociek R., Słota D., Król M., Matula G. and Kwaśny W. (2017): Modeling of heat distribution in porous aluminum using fractional differential equation.- Fractal Fract., vol.1, No.1, pp.17. doi.org/10.3390/fractalfract1010017

[6] Bekir A., Aksoy E. and Cevikel A.C. (2015): Exact solutions of nonlinear time fractional partial differential equations by sub-equation method.- Math. Methods Appl. Sci., vol.38, No.13, pp.2779-2784. https://doi.org/10.1002/mma.3260

[7] Sonmezoglu A. (2015): Exact solutions for some fractional differential equations.- Adv. Math. Phys., vol.2015, pp.567842. https://doi.org/10.1155/2015/567842

[8] Gulian M., Raissi M., Perdikaris P., Karniadakis G. and Karniadakis G. (2019): Machine learning of space-fractional differential equations.- SIAM J. Sci. Comp., vol.41, No.4, pp.2485-2509. https://doi.org/10.1137/18M1204991

[9] Esmailzadeh E., Younesian D. and Askari H. (2018): Analytical Methods in Nonlinear Oscillations.- Amsterdam: Springer.

[10] Arqub O.A., El-Ajou A. and Momani S. (2015): Constructing and predicting solitary pattern solutions for nonlinear time-fractional dispersive partial differential equations.- J. Comput. Phys., vol.293, pp.385-399. https://doi.org/10.1016/j.jcp.2014.09.034

[11] Eltayeb H., Abdalla Y.T., Bachar I. and Khabir M.H. (2019): Fractional telegraph equation and its solution by natural transform decomposition method.- Symmetry, vol.11, No.3, pp.334. https://doi.org/10.3390/sym11030334

[12] El-Ajou A., Arqub O.A. and Momani S. (2015): Approximate analytical solution of the nonlinear fractional KdVBurgers equation: a new iterative algorithm.- J. Comput. Phys., vol.293, pp.81-95. https://doi.org/10.1016/j.jcp.2014.08.004

[13] Wang K.L., Wang K.J. and He C.H. (2019): Physical insight of local fractional calculus and its application to fractional Kdv-Burgers-Kuramoto equation.- $\quad$ Fractals, vol.27, No.7, pp.1950122. https://doi.org/10.1142/S0218348X19501226

[14] Goodrich C. and Peterson A.C. (2015): Discrete Fractional Calculus.- Berlin: Springer.

[15] Li C. and Zeng F. (2015): Numerical Methods for Fractional Calculus.- Boca Raton: CRC Press, Vol. 24. 
[16] Sun H., Zhang Y., Baleanu D., Chen W. and Chen Y. (2018): A new collection of real world applications offractional calculus in science and engineering.- Comm. Nonlinear Sci. Numer. Simulat., vol.64, pp.213-231. https://doi.org/10.1016/j.cnsns.2018.04.019

[17] Hu Z. and Du X. (2015): First order reliability method for time-variant problems using series expansions.- Struct. Multidiscip. Optim., vol.51, No.1, pp.1-21. https://doi.org/10.1007/s00158-014-1132-9

[18] Turkyilmazoglu M. (2019): Accelerating the convergence of Adomian decomposition method (ADM).- J. Comput. Sci., vol.31, pp.54-59. https://doi.org/10.1016/j.jocs.2018.12.014

[19] Jajarmi A. and Baleanu D. (2020): A new iterative method for the numerical solution of high-order non-linear fractional boundary value problems.- Front. Phys., vol.8, pp.220. https://doi.org/10.3389/fphy.2020.00220

[20] Bhalekar S. and Daftardar-Gejji V. (2010): Solving evolution equations using a new iterative method.- Numer. Methods Partial Differ. Equ., vol.26, No.4, pp.906-916. https://doi.org/10.1002/num.20463

[21] Bhalekar S. and Daftardar-Gejji V. (2008): New iterative method: application to partial differential equations.Appl. Math. Comput., vol.203, No.2, pp.778-783. https://doi.org/10.1016/j.amc.2008.05.071

[22] Awawdeh F. (2010): On new iterative method for solving systems of nonlinear equations.- Numer. Algorithms, vol.54, No.3, pp.395-409. https://doi.org/10.1007/s11075-009-9342-8

[23] Bocanegra S.Y., Gil-González W. and Montoya O.D. (2020): A new iterative power flow method for ac distribution grids with radial and mesh topologies.- In: 2020 IEEE International Autumn Meeting on Power, Electronics and Computing (ROPEC) (Vol. 4). New York: IEEE. https://doi.org/10.1109/ROPEC50909.2020.9258750

[24] Qureshi S. and Yusuf A. (2019): Modeling chickenpox disease with fractional derivatives: From Caputo to Atangana-Baleanu.-Chaos Solitons Fractals, vol.122, pp.111-118. https://doi.org/10.1016/j.chaos.2019.03.020

[25] Giusti A. (2020): General fractional calculus and Prabhakar's theory.- Comm. Nonlinear Sci. Numer. Simulat., vol.83, pp.105114. https://doi.org/10.1016/j.cnsns.2019.105114

[26] Shen J.M., Rashid S., Noor M.A., Ashraf R. and Chu Y.M. (2020): Certain novel estimates within fractional calculus theory on time scales.- AIMS Math., vol.5, No.6, pp.6073-6086. https://doi.org/10.3934/math.2020390

[27] Qureshi S. and Yusuf A. (2019): Fractional derivatives applied to MSEIR problems: Comparative study with real world data.- Eur. Phys. J. Plus, vol.134, No.4, pp.171. https://doi.org/10.1140/epjp/i2019-12661-7

[28] Luchko Y. and Gorenflo R. (1998): The initial value problem for some fractional differential equations with the Caputo derivatives.- Preprint No. A-98-08.

[29] Gómez-Aguilar J.F., Yépez-Martínez H., Escobar-Jiménez R.F., Astorga-Zaragoza C.M. and Reyes-Reyes J. (2016): Analytical and numerical solutions of electrical circuits described by fractional derivatives.- Appl. Math. Model., vol.40, No.21-22, pp.9079-9094. https://doi.org/10.1016/j.apm.2016.05.041

[30] El-Ajou A., Arqub O.A., Momani S., Baleanu D. and Alsaedi A. (2015): A novel expansion iterative method for solving linear partial differential equations of fractional order.- Appl. Math. Comput., vol.257, pp.119-133. https://doi.org/10.1016/j.amc.2014.12.121

[31] Rasheed M., Shihab S., Rashid T. and Enneffati M. (2021): Some step iterative method for finding roots of a nonlinear equation.- JQCM, vol.13, No.1, pp.95-102. https://doi.org/10.29304/jqcm.2021.13.1.753

[32] Atangana A. (2016): On the new fractional derivative and application to nonlinear Fisher's reaction-diffusion equation.- Appl. Math. Comput., vol.273, pp.948-956. https://doi.org/10.1016/j.amc.2015.10.021

[33] Amat S., Busquier S. and Gutiérrez J.M. (2003): Geometric constructions of iterative functions to solve nonlinear equations.- J. Comput. Appl. Math., vol.157, No.1, pp.197-205. https://doi.org/10.1016/S0377-0427(03)00420-5

Received: March 23, 2021

Revised: July 24, 2021 